\title{
Time-resolved 3D visualization of air injection in a liquid-saturated refractive-index-matched porous medium
}

\author{
Journal Article \\ Author(s): \\ Kong, Xiang-Zhao; Holzner, Markus; Stauffer, Fritz; Kinzelbach, Wolfgang \\ Publication date: \\ 2011-06 \\ Permanent link: \\ https://doi.org/10.3929/ethz-b-000037712 \\ Rights / license: \\ In Copyright - Non-Commercial Use Permitted \\ Originally published in: \\ Experiments in Fluids 50(6), https://doi.org/10.1007/s00348-010-1018-6
}




\title{
Time-resolved 3D visualization of air injection in a liquid-saturated refractive-index-matched porous medium
}

\author{
Xiang-Zhao Kong • Markus Holzner • \\ Fritz Stauffer $\cdot$ Wolfgang Kinzelbach
}

Received: 9 March 2010/Revised: 29 November 2010/Accepted: 3 December 2010/Published online: 24 December 2010

(C) Springer-Verlag 2010

\begin{abstract}
The main goal of this work is to implement and validate a visualization method with a given temporal/ spatial resolution to obtain the dynamic three-dimensional (3D) structure of an air plume injected into a deformable liquid-saturated porous medium. The air plume develops via continuous air injection through an orifice at the bottom of a loose packing of crushed silica grains. The packing is saturated by a glycerin-water solution having the same refractive index and placed in a rectangular glass container. By using high-speed image acquisition through laser scanning, the dynamic air plume is recorded by sequential tomographic imaging. Due to the overlap between adjacent laser sheets and the light reflection, air bubbles are multiply exposed in the imaging along the scanning direction. Four image processing methods are presented for the removal of these redundant pixels arising from multiple exposure. The respective results are discussed by comparing the reconstructed air plume volume with the injected one and by evaluating the morphological consistency of the obtained air plume. After processing, a 3D dynamic air flow pattern can be obtained, allowing a quantitative analysis of the air
\end{abstract}

X.-Z. Kong · M. Holzner · F. Stauffer · W. Kinzelbach Institute of Environmental Engineering, ETH-Zurich, 8093 Zurich, Switzerland

Present Address:

X.-Z. Kong $(\square)$

Department of Geology and Geophysics,

University of Minnesota-Twin Cities,

Minneapolis, MN, USA

e-mail: xkong@umn.edu

Present Address:

M. Holzner

Max Planck Institut für Dynamik und Selbstorganisation,

Bunsenstrasse 10, 37073 Göttingen, Germany flow dynamics on pore-scale. In the present experimental configuration, the temporal resolution is $0.1 \mathrm{~s}$ and the spatial resolution is $0.17 \mathrm{~mm}$ in plane and about $1 \mathrm{~mm}$ out of plane of the laser sheet.

\section{Introduction}

The field of gas injection into a liquid-saturated porous medium is an active area of research due to its theoretical and practical applications, involving remediation (Leeson et al. 2001; Reddy et al. 1999), biological processes (Dror et al. 2004; Fry et al. 1997), aeration of filters (Canler et al. 1994; Viotti et al. 2002), marine science (Boudreau et al. 2002; Gardiner et al. 2003; Johnson et al. 2002; Paull et al. 2007), and others.

Fingering of air displacing a more viscous fluid has been extensively confirmed in Newtonian fluids (see e.g., Bensimon et al. 1986, and the references therein; see e.g., Homsy 1987, and the references therein), complex fluids (Chevalier et al. 2007, 2009; Lindner et al. 2002), and in saturated porous media (Lenormand et al. 1983) since the 1980s. Interaction of air flow and granular packing may lead to local failure of the packing and an instability, which can cause the fingers to migrate (Kong et al. 2009) and cause mobilization and compaction of the packing (Kong et al. 2010). For either air injection into loosely packed granular media (Knudsen et al. 2008) or dense granular suspensions (Chevalier et al. 2009), the forces exerted by the air interface over the granular medium mobilize the grains. According to Anderson et al. (1998), bubbles can be classified into three types: (a) bubbles that wholly fit within a pore between grains; (b) bubbles that contain several adjacent pores; and (c) bubbles that push the surrounding grains aside as they grow. A bubble of type (a) 
will first form and grow into type (b) as gas is continually injected into the medium. Until a critical pressure inside the bubble is reached, a type (b) bubble is sufficient to overcome the shear strength of the surrounding medium. The medium then fractures, expanding a type (b) bubble into a type (c) bubble and lowering the pressure. These bubbles can be easily verified in two-dimensional experiments (see e.g., Kong et al. 2009, 2010). Apparently, the pressure difference across the bubble interface is the driving force of the mobilization motion. For such a system of coupled fluid-grain flows and interactions, the situation is rather complicated, essentially due to the complexity of a granular material; it may act as a fluid or jam at high granular concentration and behave like a solid matrix. Overall, understanding the interfacial dynamic processes between the immiscible phases is crucial for revealing the underlying physical mechanisms.

As we can see in the literature, most studies of immiscible fluid-fluid displacement have focused on the behavior in two-dimensional (2D) systems, which indeed helps us to understand the underlying physics. Nevertheless, threedimensional (3D) systems are involved in most practical issues, and therefore, a corresponding detailed description of a 3D system is extremely important and required for a full characterization of the behavior. However, due to the opaqueness of porous media, the visualization of flow fields in a non-intrusive way is difficult. Therefore, only few experiments in fully 3D systems have been conducted in recent years. For example, in problems of oil recovery, X-ray computed tomography (CT) has been applied in laboratory setups. Chen et al. (1996) used this technique in their air sparging experiments. Recently, it was used in the analysis of bubble dynamics in sediments (Best et al. 2004, 2006; Boudreau et al. 2005). Nuclear magnetic resonance imaging (NMRI) is another technique that allows spatial differentiation between the phases water and air, as air yields no resonance signal in contrast to the proton spins of the water molecule (see e.g., Chen and Kinzelbach 2002; Oswald et al. 2007). The 3D NMRI is, however, slow and limited in spatial resolution to voxels on the order of $1 \mathrm{~mm}$ side-length. Moreover, the iron content of natural soils often does not allow highly resolving NMRI, as in the high magnetic fields, artifacts are introducing too much disturbance into the image. An alternative in this case is the use of neutron scattering. In neutron scattering, the protons in the water molecule are scattering the incident low-energy neutrons from a reactor. Water is therefore more opaque in the image while air and the medium stay transparent. The temporal resolution of the method, though better than in NMRI, is still low. The spatial resolution is comparable to that of NMRI.

An alternative method exists, which follows the principle of light transmission in an optically homogeneous medium. It uses transparent granular materials saturated with a liquid of the same refractive index and eventually combining it with a laser-induced fluorescence technique. With this method, the difficulty of accessing the information of displacement in the opaque porous media can be overcome to a certain degree. Walmann (1992) used this method to observe a dispersion structure of miscible tracer molecules in porous media. Montemagno and Gray (1995) applied the photoluminescent volumetric imaging to visualize the multiphase flow and transport in a porous medium. Rashidi et al. (1996) quantitatively measured the pore geometry, fluid velocity, and solute concentration within a saturated 3D porous medium. Fontenot and Vigil (2002) visualized the non-aqueous phase liquid dissolution and fragmentation events in porous media. A similar technique has been applied to visualize the imbibition of water into an oil-filled porous medium, the transport of a fluorescent dye (Stöhr et al. 2003), and the drainage displacement under combined capillary and gravity effects in indexmatched porous media (Ovdat and Berkowitz 2006).

In order to quantitatively analyze the displacement patterns, a 3D data set must be extracted. This problem can be solved by subdividing the observation volume into a sequence of $2 \mathrm{D}$ slices, which then is converted into a $3 \mathrm{D}$ volume data set. This scanning concept is commonly used in tomography and is also well known in the whole-field velocity measurements (see e.g., Brücker 1996, 1997; Hoyer et al. 2005). The important parameters are slice thickness, maximum scan volume, overall achievable volume scan rate with a given camera, and slice location.

The current investigation studies a non-wetting fluid (air) displacing a wetting fluid (liquid) inside a non-consolidated granular packing, with the non-wetting fluid being injected at the center of the 3D box' bottom via an orifice. Due to the fact that the invading air is much less dense and much less viscous than the displaced liquid, the displacement pattern is affected by both the viscous and density instabilities. Furthermore, the current study also considers the deformation of the medium, which is an unconsolidated granular packing placed randomly in a $200 \mathrm{~mm}$ (width) $\times 200 \mathrm{~mm}$ (depth) $\times 300 \mathrm{~mm}$ (height) glass container.

The task of this work is to establish a method to properly obtain the dynamic 3D structure of the injected air plume. Although 3D reconstruction from 2D slices is a welldeveloped procedure, e.g. in medical science, challenges arise, when the application of this technique concerns a dynamic process like the air injection investigated herein. The great advantage of the presented method is the flexible temporal resolution. The paper is organized as follows. After a detailed description of the experimental setup in Sect. 2, the presentation and discussion of the results will be given in Sect. 3 The experimental and processing methods are verified through measurements of the plume 
volume and comparison of the obtained shapes of the plume structure. Conclusions are given in Sect. 4.

\section{Experimental setup and methods}

\subsection{Materials}

The experimental principle here is based on the use of granular materials and wetting liquid that have the same refractive index, making the system transparent. Theoretically, the refractive index of two materials matches at one wavelength $\lambda$. In an optically homogeneous system composed of granular materials and a matched liquid, only the light with this wavelength $\lambda$ can be transmitted in a straight line. The paths of rays lying in other parts of the spectrum are distorted. Here, the refractive index of the fused silica glass (the granular materials) is $n=1.457$ for a blue-light laser $(\lambda \simeq 490 \mathrm{~nm})$.

The porous matrix is constructed by crushed fused silica glass grains with a density of $\rho_{s}=2,202 \mathrm{~kg} / \mathrm{m}^{3}$. Two different grain size distributions have been tested in the current study, $d_{1}=1.0-1.6 \mathrm{~mm}$ and $d_{2}=0.6-0.8 \mathrm{~mm}$. In order to match the refractive index of the grains, the defending wetting fluid used in the experiments is a $90-10 \%$ by volume glycerinwater solution. The wetting fluid has a dynamic viscosity of $\mu_{l}=0.165 \mathrm{Pas}$ and a density of $\rho_{l}=1,235 \mathrm{~kg} / \mathrm{m}^{3}$ at room temperature. Air is used as the invading non-wetting fluid. The corresponding parameters for air are $\mu_{a}=1.83 \times$ $10^{-5} \mathrm{~Pa} \mathrm{~s}$ and $\rho_{a}=1.2 \mathrm{~kg} / \mathrm{m}^{3}$. The surface tension between these two fluids is $\sigma=6.4 \times 10^{-2} \mathrm{~N} / \mathrm{m}$.

Assemblies of liquid-saturated grain packings were randomly placed inside a 3D glass container of the size $200 \mathrm{~mm}$ (width) $\times 200 \mathrm{~mm}$ (depth) $\times 300 \mathrm{~mm}$ (height). The global porosity was measured to be on average $\phi=0.48$. An orifice with a diameter of $0.3 \mathrm{~mm}$ at the central point of the base serves as the injection point of the air flow rate $Q$. The orifice was installed to diminish the pressure fluctuation produced by the fluctuating instability of bubbles forming and breaking at the orifice. This technique also constrains the feedback of the air flow in the system on the injection. The flow rate is measured using a GSM-A5TA-BN00 flow-meter. It has an accuracy of $\pm 1.5 \%$ and a repeatability of $\pm 0.1 \%$ a of full-scale measurement.

In all experiments, the matrix has an initial height of $H_{b}=118 \pm 2 \mathrm{~mm}$. The supernatant fluid has a constant surface elevation at $H_{w}=195 \mathrm{~mm}$ above the base of the box. Before starting an experiment, the system was vibrated for a few minutes, guaranteeing that a random porous matrix was packed homogeneously under gravity. In this paper, we show the results of four representative experiments out of a total of 25 . Two experiments with grain size $d_{1}$ and air injection rates of $16.4 \mathrm{ml} / \mathrm{min}$ ("Exp. 1") and $41.1 \mathrm{ml} / \mathrm{min}$ ("Exp. 2") are presented in detail to compare the different image processing methods. Two experiments with grain size $d_{2}$ and air injection rates $8.0 \mathrm{ml} / \mathrm{min}$ ("Exp. 3 ") and $141.7 \mathrm{ml} / \mathrm{min}$ ("Exp. 4") are used to investigate the possible effects of the grain size on the results.

\subsection{Apparatus}

In order to visualize the distribution of the air phase, a recording system with a high-speed camera and a laser scanning illumination system are employed. Figure 1 illustrates the current experimental setup for the 3D system. The light source for illumination is a continuous $20-\mathrm{W}$ argon ion laser. The laser beam has a wavelength of $\lambda \simeq$ $490 \mathrm{~nm}$ (after passing a blue dichroic filter produced by Edmund Industrial Optics) and a diameter of around $2.0 \mathrm{~mm}$. A series of lenses comprising a cylindrical planoconvex lens, a plano-concave, and then again a cylindrical plano-convex lens spread the beam to a sheet of a "static" thickness of about $0.3 \mathrm{~mm}$. Following this set of lenses, the vertical light sheet passes through an octagonal Plexiglas cylinder (refractive index $n_{c}=1.49$ ) composed of eight prisms. This cylinder can rotate at a set speed around its axis while the light sheet passes through the parallel facing planes of the cylinder. The octagonal Plexiglas cylinder produces a continuous scanning motion of the light sheet. It is flexible with respect to the total thickness of each slice since the control of the total thickness is accomplished through the camera timing, as will be described in more detail below. However, the maximum sweep of the light sheet is determined by the geometry of the octagonal cylinder (as shown in Fig. 2). The octagonal cylinder axis is mounted vertically and aligned carefully with the incoming light sheet. By its rotation, the angle of incidence, $\theta$, of the incoming light sheet varies with $\dot{\theta}=2 \pi f_{v} \mathrm{rad} / \mathrm{s}$ and is

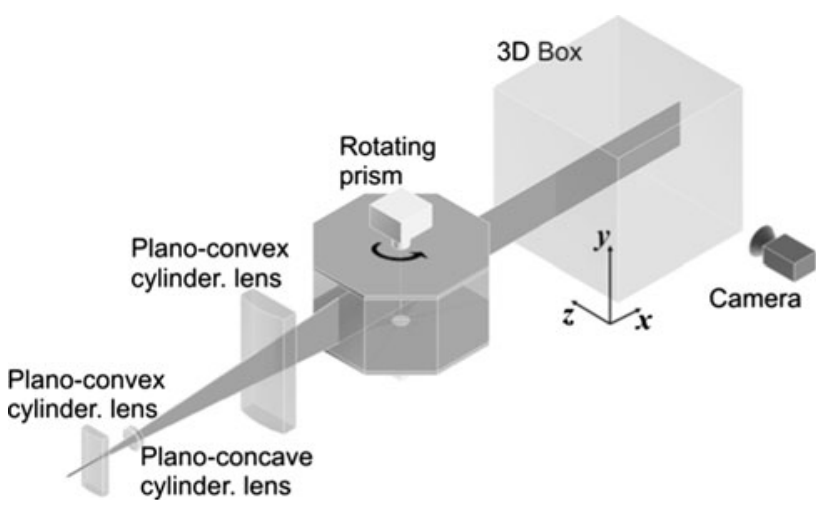

Fig. 1 Sketch of the 3D experimental setup. The laser beam is expanded and thinned to a light sheet and then scanned through the observation volume using an eight-face prism. The coordinate system $x-y-z$ of the experiments is also shown 


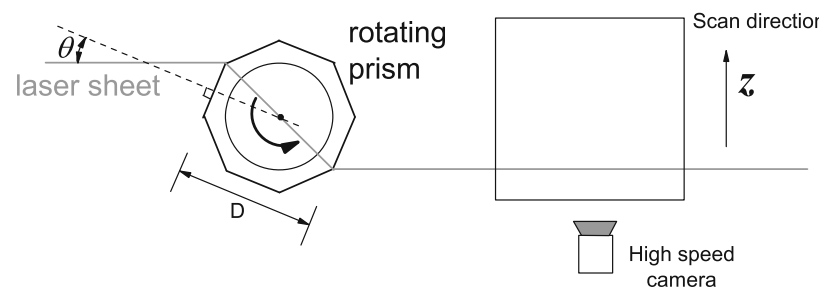

Fig. 2 Sketch of the top view of the experimental setup. Here, $\theta$ is the angle of incidence and $z$ is the coordinate parallel to the scanning direction and in line with the symmetry axis of the camera arrangement. $D$ is the distance between two parallel faces of the octagon (the inner circle diameter of the octagonal cylinder)

limited by $-\pi / 8$ to $\pi / 8$ due to the octagonal shape of the cylinder, where $f_{v}$ is the volume scan frequency. Each time the limiting angle is passed, the incident light sheet enters through the next face of the octagon, starting at a new angle shifted by $\pi / 4$. For each eighth of a rotation of the octagonal cylinder, the $z$-coordinate of the exiting light sheet shifts from -24 to $24 \mathrm{~mm}$, where $z$ is the coordinate along the scanning direction, coinciding with the symmetry axis of the camera arrangement (as shown in Fig. 2).The relation of $z$ to the other geometric parameters is shown in Fig. 3, which gives $z=\left(l^{*}-l\right) \cos \theta$, where $l^{*}=D \tan \theta$, $l=D \tan \theta_{c}$, and $n_{c} \sin \theta_{c}=n_{a} \sin \theta$ (Snell's Law), yielding the parallel shift,

$z=D \sin \theta\left[1-\frac{\cos \theta}{\sqrt{\left(\frac{n_{c}}{n_{a}}\right)^{2}-\sin ^{2} \theta}}\right]$,

where $D=175 \mathrm{~mm}$ is the inner circle diameter of the octagonal cylinder. The parallel shift $z$ is used in the determination of the slice position in later image processing (3D reconstruction). As $\theta$ varies from $-\pi / 8$ to $\pi / 8, z$ is approximately a linear function of $\theta$ (as shown in Fig. 4). Thus, achieve a maximum frame the slice position is assumed to vary linearly with $\theta$ in later image processing.

Through this scanning technique, the observation volume is subdivided into a series of slices. The slices were recorded by a high-speed digital camera (Photron Ultima APX) that is capable to achieve a maximum frame rate of

Fig. 3 Principle of parallel shift when light is transmitted between two parallel faces of the octagon. $n_{a}$ is the refractive index of air. $\theta_{c}$ is the angle of refraction. $l$ is the horizontal displacement when the light reaches the opposite side of the octagon. $l^{*}$ is the virtual horizontal displacement when the light would reach the opposite side without refraction

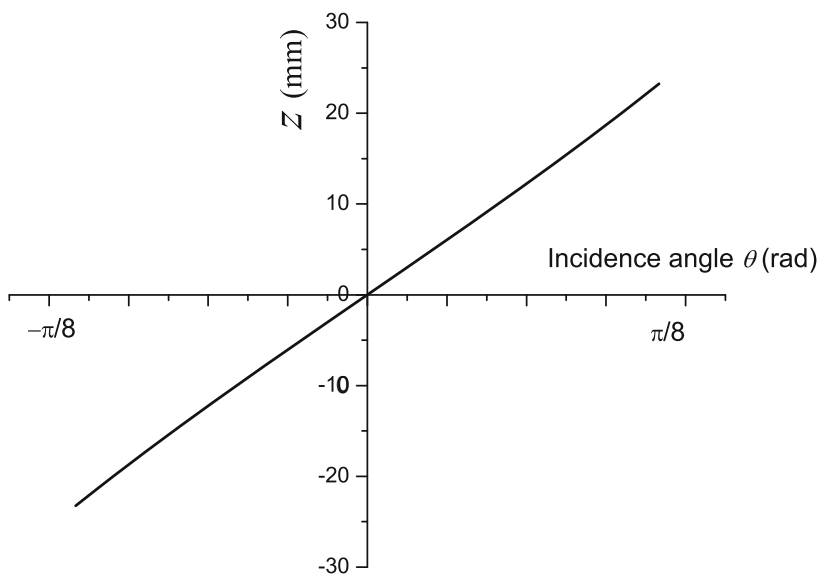

Fig. 4 According to Eq. 1, the shift distance $z$ depends on the angle of incidence $\theta$

$2 \mathrm{kHz}$ at a full resolution of $1,024 \times 1,024$ pixels. With a built-in physical memory of $6 \mathrm{~GB}$, the system is able to record a time series of 6,000 images. A Nikkor Micro $60 \mathrm{~mm}$ lens with an aperture of $f 32$ allows a good focus over the entire scanning depth of $50 \mathrm{~mm}$. The recording frame rate $f_{r}$ is related to the volume scanning rate $f_{v}$ by the number of slices $n_{s}$ :

$f_{r} \geq n_{s} f_{v}$.

This means that, for a given $f_{r}$, one can either choose small $n_{s}$ and large $f_{v}$ or large $n_{s}$ and small $f_{v}$, thereby increasing the spatial resolution by lowering the time resolution or vice versa. The exposure time is set to be the inverse of the frame rate. Therefore, the camera always integrates over the exposure time along the path of the moving light sheet. This means that the total thickness of one single slice depends on the "static" thickness of the light sheet and the "dynamic" thickness, i.e., sweep distance during the exposure time. Figure 5 shows that the sweep distance $\Delta z$ can be calculated as $\Delta z=v / f_{r}$, where $v$ is the scanning velocity. The scanning velocity in the experiments is around $50 \mathrm{~cm} / \mathrm{s}$ with a volume scan rate of $f_{v}=10 \mathrm{~Hz}$. From Fig. 5, it can be seen that between two adjacent slices, there is an overlap distance of $\delta z_{p}$ which is around $0.5 \mathrm{~mm}$.

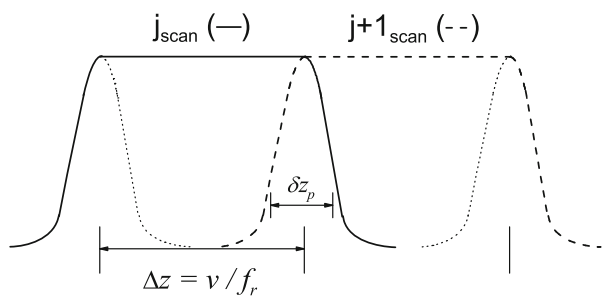

Fig. 5 Schematic illustration of the light sheet intensity distribution during scanning with the velocity $v$ 


\subsection{Illumination principle}

By refining the index-matching technique and measurement resolution in our current method, the contrast between air (refractive index $n=1.0$ ) and the background (refractive index $n=1.457$ ) is so strong that the interface between air phase and the medium reflects enough light to visualize the invading structure. During most of the experiments, the structure invades the pores between the grains, thus the interface is rough. As a result, the reflection from the interface is diffuse, making the whole interface visible in the camera. Since we have an unconsolidated packing, at the later stage of the experiment, the grains are pushed aside by the air bubbles, and therefore, there is a sharp transition to a behavior that is similar to a bubble in a liquid. In such a case, the interface is rather smooth, making the reflection specular. Therefore, only parts of the interface are visible (see e.g., Honkanen 2006), and the performance of our method decreases, as will be discussed in the section below.

In $x$-direction, however, a measurable divergence of the light sheet was detected by measuring the change of thickness of the sheet. After traversing through the well-matched medium, the sheet has a variation in thickness from 0.3 to $0.8 \mathrm{~mm}$. Since the air plume develops around the middle of the box, we take the light sheet thickness at the middle of the field of view as the representative thickness, which is approximately $0.5 \mathrm{~mm}$. In this sense, we assume that the light intensity is approximately uniform when the laser sheet passes through the field of interest in the saturated medium. However, as the laser sheet crosses the injected air plume, light is reflected and refracted at the plume surface and one would therefore expect that the light intensity in the sheet should somewhat attenuate in $x$-direction. In preliminary experiments, we did not detect a systematic attenuation of the measured grayvalue intensity in $x$-direction even when the local number density of air bubbles was high. As it will be shown below, the reconstructed plume structures present a well-balanced shape, i.e., without the loss of information in $x$-direction.

In the present setup, with a fixed recording frame rate of $f_{r}=500 \mathrm{~Hz}$, each volume scan consists of a maximum of 50 illuminated light sheets. Therefore, we have a total thickness of one single slice of $\Delta z+\delta z_{p}=1.5 \mathrm{~mm}$ and an overlap distance of $\delta z_{p}=0.5 \mathrm{~mm}$ between two adjacent slices. Considering that 50 slices are evenly placed over a distance of $50 \mathrm{~mm}$ (Fig. 4), the spacing between the slices is $1 \mathrm{~mm}$. In the present configuration, the observed volume has dimensions of about $85 \times 120 \times 50 \mathrm{~mm}^{3}$.

\section{Results}

In this section, we present the results of four representative experiments, which were carried out with air continuously injected into the porous medium of grain size $d_{1}($ Exp. 1, 2) and $d_{2}$ (Exp. 3, 4), at approximate constant injection rates. Before coming to these results, we show a preliminary measurement obtained from a static state, where the bubbles were stably trapped in the porous medium consisting of the grain $d_{1}$. The static state was obtained by stopping the injection after breakthrough and keeping the system at rest for one day. Thereafter, the "frozen" structure of the entrapped air was measured via the scanning method and visualized. The left-hand-side of Fig. 6 shows a photograph of the structure using a normal digital camera at room illumination. For comparison, a 3D reconstruction is shown on the right. For this reconstruction, the grayvalue images were first transformed into binary format, i.e., all pixels with a non-zero grayvalue are set to 1 and then visualized using the isosurface function in Matlab. From the figure, we note that on the qualitative level, all the important features of the structure can be satisfactorily reproduced without any further processing.

However, the structures appear unnaturally elongated in $z$-direction. As an illustrative example, in Fig. 7, we depict a sequence of grayvalue images (top) of a small air bubble with a diameter of less than $1 \mathrm{~mm}$ and associated contour slices (bottom). It can be seen that the maximum exposure is realized in slice $\mathrm{nr} .4$ while with smaller grayvalues, the image of the bubble is still well visible in two neighboring slices on each side. Ideally, this bubble should only be visible in slice $\mathrm{nr}$. 4. As mentioned in the previous section, the light sheets overlap with each other and this leads to the redundant bubble images over several consecutive slices (Fig. 7). Due to the finite thickness of the light sheet, the overlap effect leads to redundant exposures in at most two neighboring slices on each side, which is the exact case shown in Fig. 7. It turned out that this is not the only effect
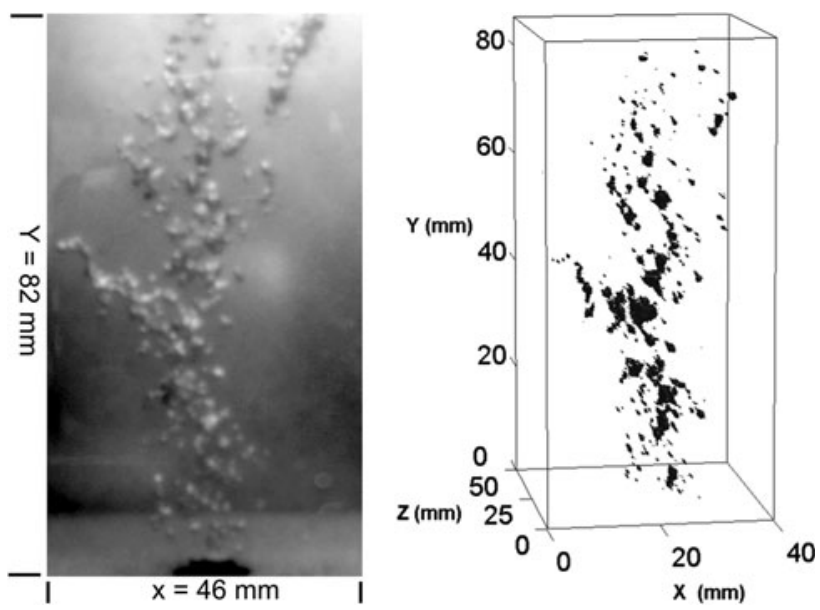

Fig. 6 Morphological consistency is verified in the case of discrete static bubbles. Left 2D image of $y-x$ panel using a normal digital camera. Right 3D reconstruction based on the raw images 

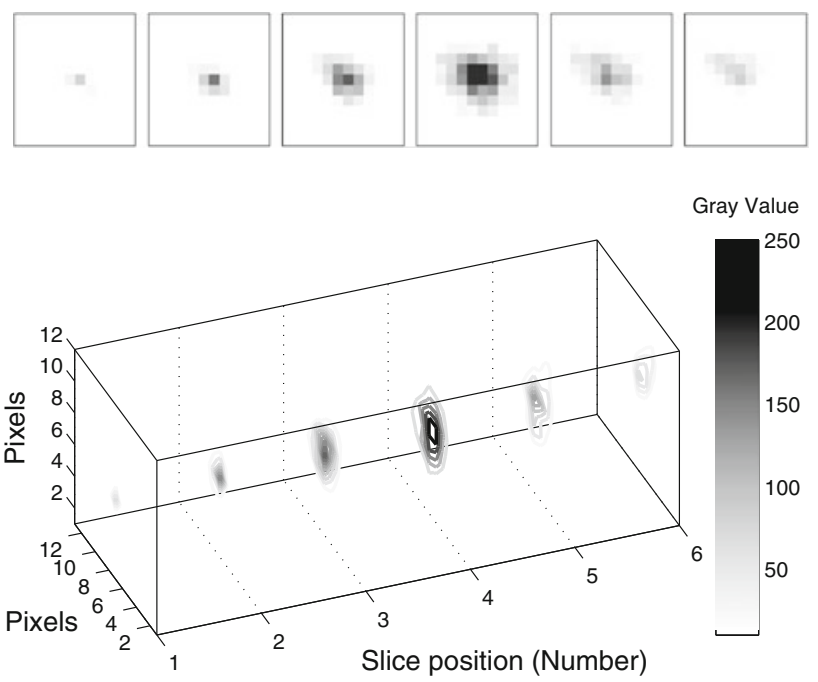

Fig. 7 Sequence of images of a bubble with a diameter smaller than $1 \mathrm{~mm}$. Top raw gray images (12 pixels $\times 13$ pixels) sequentially registering the bubble. Bottom contours of the slices, where the distance between the center of two neighboring slices is $1 \mathrm{~mm}$

causing the unnatural elongation, hereinafter referred to as multiple exposure. There is a second effect, namely air bubbles that are located out of plane of the slice can be illuminated indirectly. This indirect illumination comes from light reflected by neighboring air bubbles that are themselves directly exposed to the light sheet. This effect appears when the local number density of air bubbles is high. It can sometimes lead to structures that appear extremely elongated in the scanning direction. Typically, the grayvalue intensity of the bubble image due to indirect illumination is much lower than the consecutive exposures caused by the direct one, respectively. Precisely this feature will be used for the removal of the redundant exposures, as will be described in more detail in the following.

Due to multiply imaged bubbles, any quantitative measure like the volume or the shape of the structure in slice direction will be biased. Thus, an important pre-step in determining the invading structures is the choice of the methods used to identify and reconstruct their morphology properly. These methods are then verified by comparing the calculated reconstructed air volume with the injected one. In the following, we present and test four methods on the data obtained from Exp. 1 and Exp. 2: a level-based method where a threshold on the grayvalues intensity is used, an erosion method, a "two-step" method, and a "curvature" method based on the curvature of the grayvalue profile in the scanning direction.

From Fig. 7, we learn that a small threshold on the grayvalue intensity will help to remove the redundant pixels, while the main bubble image in slice nr. 4 will not change very much. This level-based method, i.e., based on a threshold on the grayvalue intensity, is well known in image processing. In order to proceed, a reasonable choice of the threshold value has to be made, keeping in mind that the experiments are transient. This choice might be based on a statistical measure such as the average grayvalue of the image. Figure 8 shows the variation of the average grayvalue, 〈grayvalue $\rangle$, in time for the two dynamic experiments, where the angle brackets denote the average of all non-zero grayvalues in space, i.e., averaged both over the image plane and over 50 images belonging to a given volume scan. From Fig. 8, we note that $\langle$ grayvalue $\rangle$ varies in time, and therefore, we apply a time-dependent threshold. We can also see that there are some variations between the experiments. A closer inspection revealed that this is due to a higher proportion of saturated pixels, i.e., pixels with a maximum grayvalue of 255 . This is presumably due to the fact that the laser intensity also has an approximately Gaussian distribution in $y$-direction. As the plume structure grows in time, more bubbles are therefore exposed to higher light intensity.

The choice of the threshold is validated by comparing the injected volume with the one measured from the reconstructed plume structure after thresholding the images. The volume is calculated from the plume structure obtained as described above, with the addition that in a prestep, all pixels smaller than the threshold are set to zero. The result is shown in Fig. 9 for the two experiments, which shows that the volume increases monotonically in time until a maximum is reached and thereafter it decreases to a plateau. One notes that the main trend of the initial increase is in agreement with the profile of the injected volume. The injected volume is obtained through integration of the recorded flow rates over time. Also, the quantitative agreement between the two curves is generally satisfactory, with somewhat higher deviations notably in

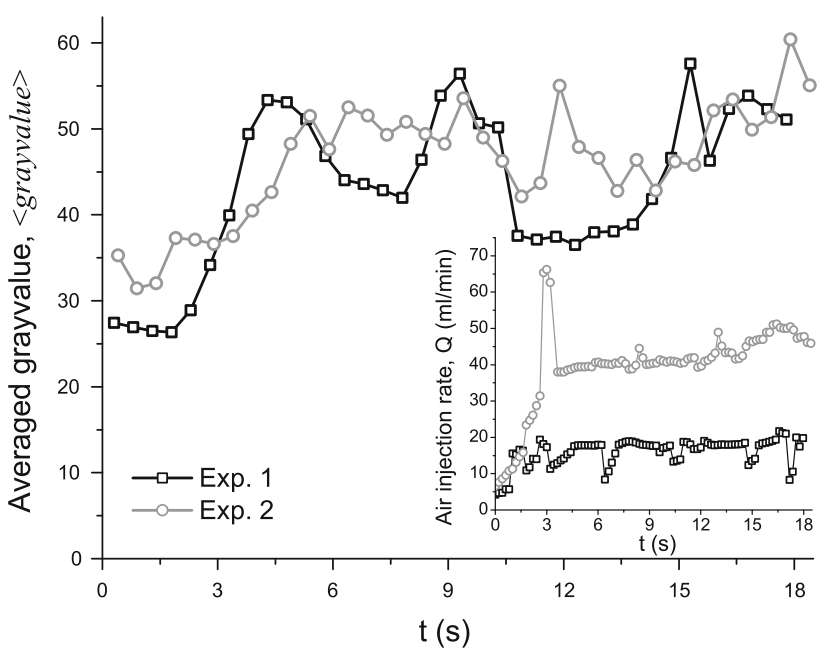

Fig. 8 Averaged grayvalue (termed $\langle$ grayvalue $\rangle$ ) in time for the two experiments. Insert: the recorded injection rates in time 


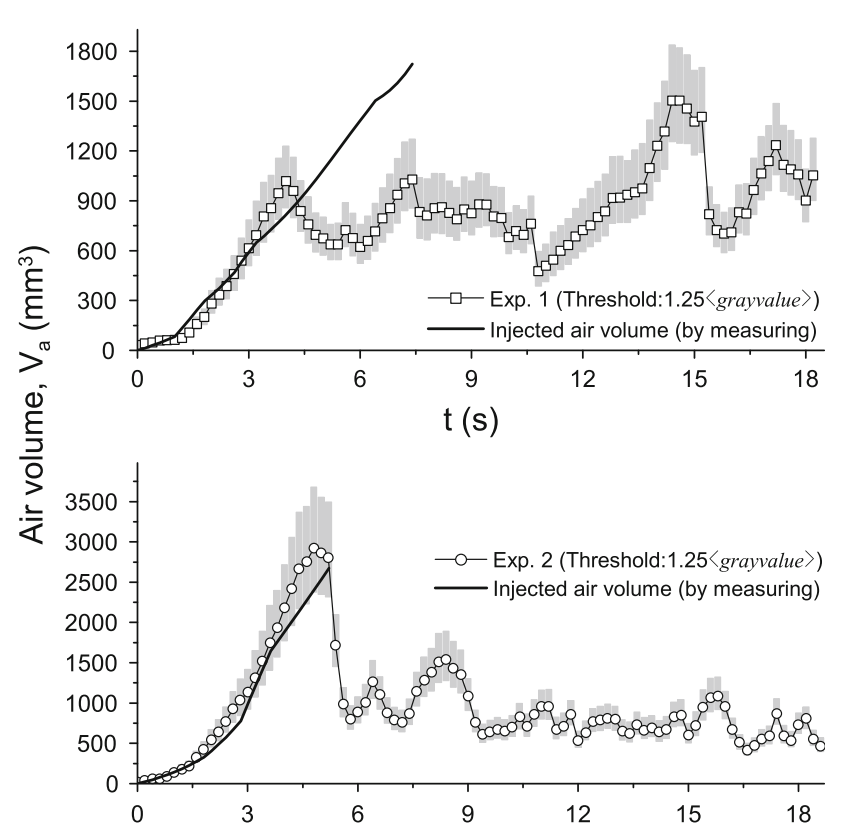

Fig. 9 Comparison of the injected air volume (continuous line) with the volume calculated from the reconstructed plume structure over time using the level-based method, where the symbols refer to the selected threshold of 1.25 〈grayvalue $\rangle$ and the shaded columns represent a variation of the threshold by \pm 0.25 〈grayvalue $\rangle$

the early time of the experiment with the injection rate of $41.1 \mathrm{ml} / \mathrm{min}$. The comparison is valid during the period when the structure remains within the limits of the field of view until breakthrough. For the two experiments, breakthrough occurs at $t=7.5 \mathrm{~s}$ and $t=5.4 \mathrm{~s}$, respectively, for
$Q=16.4$ and $41.1 \mathrm{ml} / \mathrm{min}$. The first relative maximum of the curve in Fig. 9(bottom) roughly corresponds to the breakthrough time, whereas in the upper panel, the second relative maximum of the curve corresponds to breakthrough. This means between the two relative maxima of Exp. 1, volume is lost. This can be attributed to illumination in the sense that the interface of the plume in the proximity of the top of the packing became smooth and was therefore only partly visible. For Exp. 2, there is a similar qualitative change of the interface. However, it occurs just at the breakthrough and is therefore not noticeable in the figure. Now, we analyze how this method modifies the shape of the structure.

Figure 10 depicts the reconstructions of the experiment with $Q=41.1 \mathrm{ml} / \mathrm{min}$ for a given time and from two different views, namely the front and side views. The raw data are shown in Fig. 10a, f, and the data after applying the level-based method on grayvalue intensity are shown in Fig. 10b, g. Apparently, the main features of the shape are conserved, even if many small clusters are erased. However, some elongated streaks are still clearly visible in the side view (Fig. 10g). It appears that the problem of multiple exposure is difficult to solve perfectly by using the level-based method on grayvalue intensity alone.

Another well-known image processing method is the socalled image erosion. This method takes the shape of objects into account only, irrespective of their grayvalue. Specifically, the procedure removes pixels on object boundaries of the structure. The number of pixels removed
Fig. 10 3D reconstruction of the plume structure for the experiment with injection rate $Q=41.1 \mathrm{ml} / \mathrm{min}$ at time $t=4 \mathrm{~s}$, where the upper and lower panels show the front and side view, respectively: raw data $(\mathbf{a}, \mathbf{f})$, level-based method $(\mathbf{b}, \mathbf{g})$, erosion method (c, h), "two-step" method (d, i), and "curvature" method (e, j) (a)

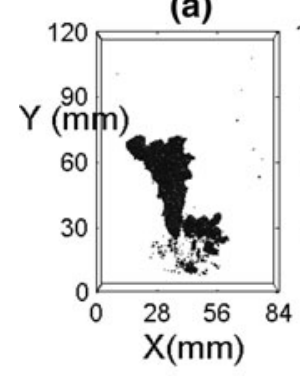

(f)

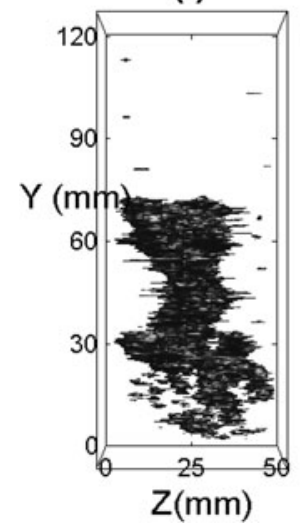

(b)

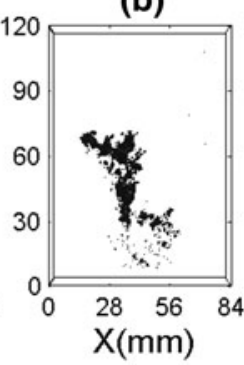

(g)

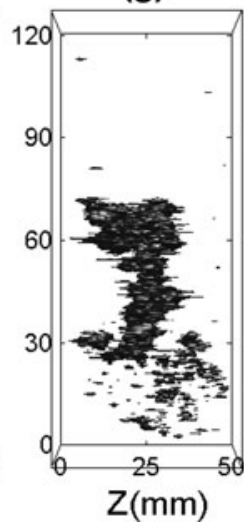

(c)

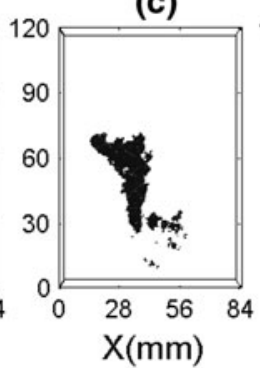

(h)

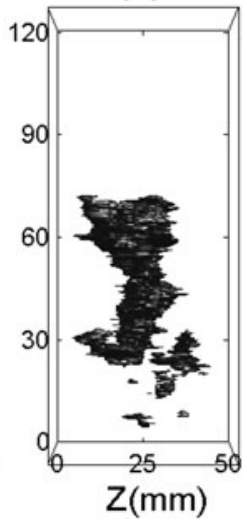

(d)

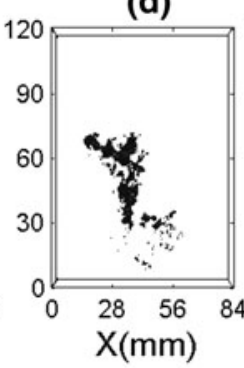

(i)

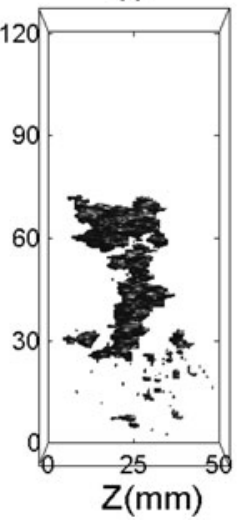

(e)

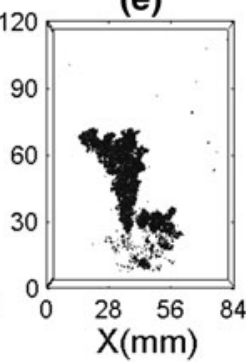

(j)

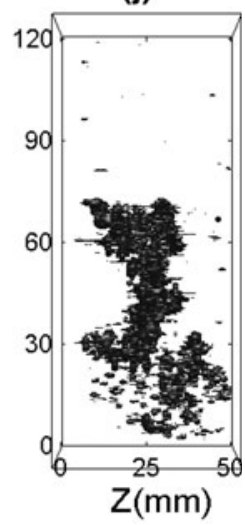


from the objects depends on the size and shape of a structuring element used to process the data. The structuring element is a matrix, which in general consists of 0's and 1's and that can have any arbitrary shape and size. We considered the simplest form of the matrix, which is a square when a $2 \mathrm{D}$ image is considered, or a cubic matrix for our 3D structure and the matrix consists of 1's only. The side-length of the structuring element is the free parameter to be specified. We applied the erosion procedure to the raw structure (Fig. 10a, f). Figure 10c, h show the front and the side views of a snapshot of the eroded plume structure, where the side-length of the structuring element is 5. In order to test the sensitivity of the result on this parameter, elements of sizes of 3 and 7 were also tried and the corresponding volume calculations are represented by the shading in Fig. 11. With the former choice, almost no effect on the plume was discernable, and with the latter choice, nearly the whole structure was erased. A choice of a side-length of 5 also makes sense if we recall the Fig. 7, where it is visible that the object is multiply imaged on two neighboring slices on either side of the object. From the side view (Fig. 10h), we see that, compared to the levelbased method (Fig. 10g), the elements of the structure are much less elongated, and therefore, the method seems to be more effective in correcting for the elongated streaks.

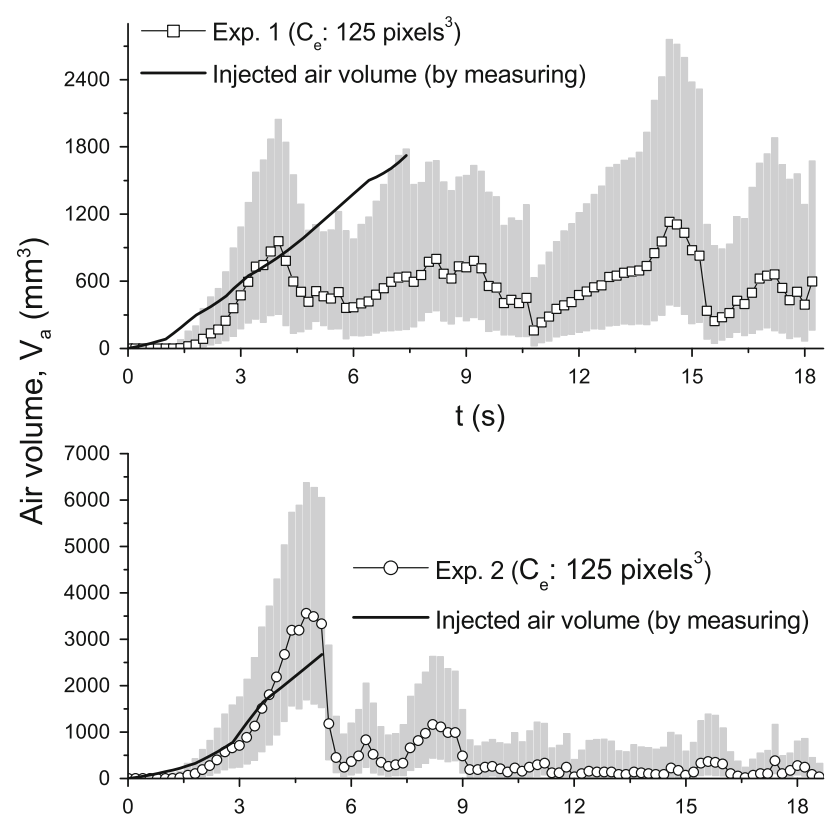

Fig. 11 Comparison of the injected air volume (continuous line) with the volume calculated from the eroded plume structure over time using the erosion method, where the symbols refer to the selected structuring element of $C_{e}\left(5 \times 5 \times 5\right.$ pixels $\left.^{3}\right)$ and the shaded columns represent a variation of the side-length by \pm 2
Figure 11 shows the comparison between the injected air volume and the one calculated from the eroded plume structure. Again, the main trend is correctly reproduced; however, quantitatively the agreement between the two curves is not as good as the one obtained via the levelbased method. After the peak, we note again a decrease in the volume until it levels off. The volume in this last stage is unexpectedly low and can become close to zero. This very low volume is in disagreement with visual observation. It might be due to the fact that the plume structure becomes somewhat more fragmented in final stages of the experiment. A more fragmented structure exposes larger boundary surfaces and is therefore more strongly eroded. So the erosion corrects efficiently for the elongated streaks, but does not work satisfactorily when the structure becomes more fragmented.

A third alternative method comes to mind by looking at Fig. 7 again that subsequent exposures of the small bubble appear not only with smaller grayvalue but also with smaller size. The multiple exposure results in elongated objects that appear as streaks on Fig. 10e and small, i.e., typically smaller than a pore size, patches in the front view Fig. 10a. It seems therefore reasonable to think of a criterion that controls the patch size on the image of a slice. It is worthwhile to keep objects that have a size on the order or larger of half an average pore size, which is about half a millimeter. On the image plane, this corresponds to an area of the patch of roughly $A_{p}=4 \times 4$ pixels $^{2}$. This approach, in the following termed "two-step" method, is now applied as a step subsequent to the above level-based method. The procedure is that, first, a grayvalue threshold is applied using a lower level compared to the one proposed above, and second, patches on the image with an area smaller than $A_{p}$ are erased. Figure 10d, i depict the reconstructions of the plume structure after applying this approach, where the selected threshold is 1.25 〈grayvalue $\rangle$. Comparing Fig. 10b with $\mathrm{d}$, we note that the two are almost identical, besides a few tiny objects. Comparing the side views Fig. $10 \mathrm{~g}$, i, it appears that the streaks associated with multiple exposures were efficiently removed. Additionally, the shape of the structure in Fig. 10i seems more realistic than the eroded structure, because the erosion method produces a more coherent structure due to the removal of smaller detached objects at the outer boundary of the plume (Fig. 10h). The comparison between the volume calculated from the reconstructed plume structure and the injected volume is shown in Fig. 12, and it looks quite similar to Fig. 9. The agreement between the two curves is overall better than in Figs. 9 and 11. However, a drawback of this method is that the reconstructed plume appears too strongly fragmented. Some connections between the parts of the plume seem to be artificially removed. Since the "two-step" method is 


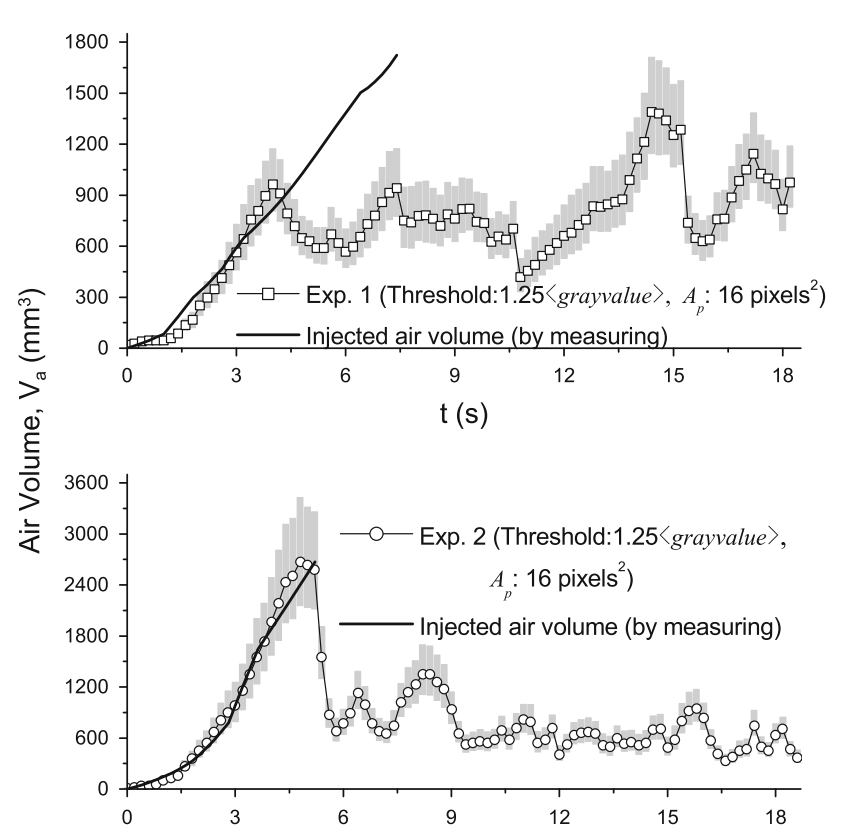

Fig. 12 Comparison of the injected air volume (continuous line) with the volume calculated from the reconstructed plume structure over time using the "two-step" method, where the symbols refer to the selected threshold of 1.25 〈grayvalue $\rangle$ and the shaded area represents a variation of the threshold by \pm 0.25 〈grayvalue $\rangle$

based on a fixed $A_{p}$, the redundant pixels are more strongly erased for small structures than for the larger ones. This is why there is an underestimation at the very beginning and overestimation near the first peak of the volume profile (Fig. 12), respectively.

Up to now, we used a threshold on the grayvalue intensity and did not consider its distribution in space. As mentioned above, the grayvalue intensity of a bubble image is high when directly exposed to the laser light (Fig. 7). Subsequent exposures due to overlapping slices or indirect illumination leave a particle image with comparatively lower grayvalue intensities. It is therefore possible to use a criterion based on the grayvalue profile in scanning direction to distinguish between the two. We found that the grayvalue curvature in scanning direction shows strongly negative values in its second derivative, i.e., a marked concave curvature, when the bubble is directly exposed to the laser light, whereas it attenuates to less negative or positive curvature values in the case of subsequent exposures. For the example of the small bubble shown in Fig. 7 , only the image of slice nr. 4 corresponding to the local maximum of the grayvalue in the scanning direction should remain and the neighboring images should be removed. By knowing the second derivative of the grayvalue in $z$-direction, one can easily remove the multiple exposures. This method is termed "curvature" method.

Let $S(x, y, z)$ be the raw data matrix that contains the grayvalues and $G_{z z}(x, y, z)$ its second derivative in $z$ - direction. By applying a central difference approximation, $G_{z z}(x, y, z)$ is given by

$G_{z z}(x, y, z)=\frac{S(x, y, z+h)-2 S(x, y, z)+S(x, y, z-h)}{h^{2}}$,

where the spacing is $h=1 \mathrm{~mm}$. This means we only consider three consecutive slices. For the exceptional case, when over three consecutive slices, the maximum grayvalue is reached, and $G_{z z}(x, y, z)$ is zero identically, these pixels are excluded from data reduction. In order to proceed, a reasonable choice of the threshold value on $G_{z z}(x, y, z)$ is needed. Considering that there might be isolated noise pixels in very low grayvalue intensity and that the experiments are transient, similar to the grayvalue threshold method described above, we use a statistical measure based on the mean in space of the negative part of $G_{z z}(x, y, z)$. Figure 13 shows the variation of the average $\left\langle G_{z z}<0\right\rangle$ in time for the two dynamic experiments, where $\left\langle G_{z z}<0\right\rangle$ was obtained by averaging the negative part of $G_{z z}(x, y, z)$ from 50 slices belonging to a given volume scan. One can see that $\left\langle G_{z z}<0\right\rangle$ varies in time and between the experiments. Similarly to the level-based method, we apply a threshold that is proportional to $\left\langle G_{z z}<0\right\rangle$. For the thresholding, a binary matrix $T(x, y, z)$ is defined as

$T(x, y, z)=\left\{\begin{array}{ll}1 & \text { if } G_{z z} \leq c\left\langle G_{z z}<0\right\rangle \\ 0 & \text { otherwise }\end{array}\right.$,

where $c$ is a positive constant. This matrix $T(x, y, z)$ is then multiplied element by element with $S(x, y, z)$.

Figure 14 shows the comparison between the injected air volume and the one calculated from the remaining structures after the "curvature" method was applied. The

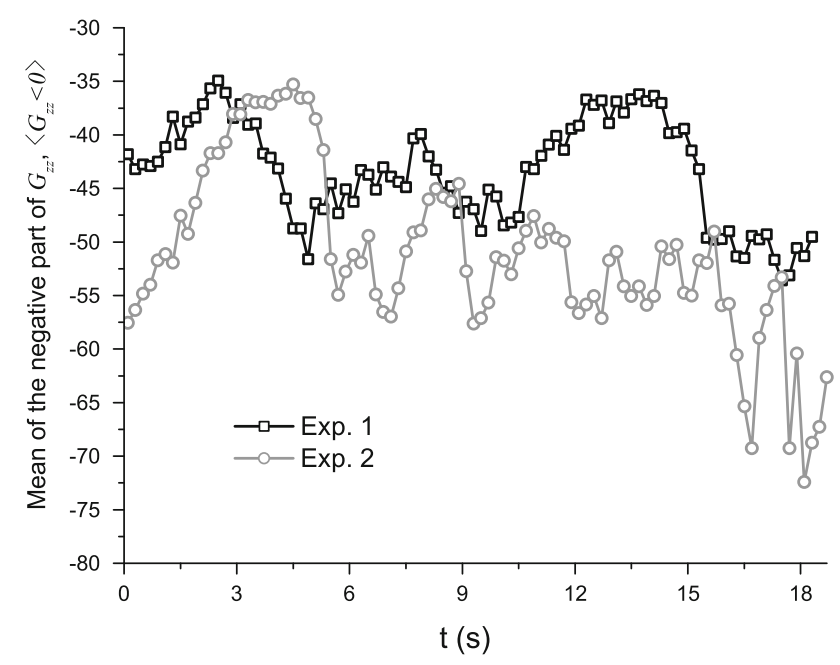

Fig. 13 Mean of the negative part of $G_{z z}$ (termed $\left\langle G_{z z}<0\right\rangle$ ) in time for the two experiments 


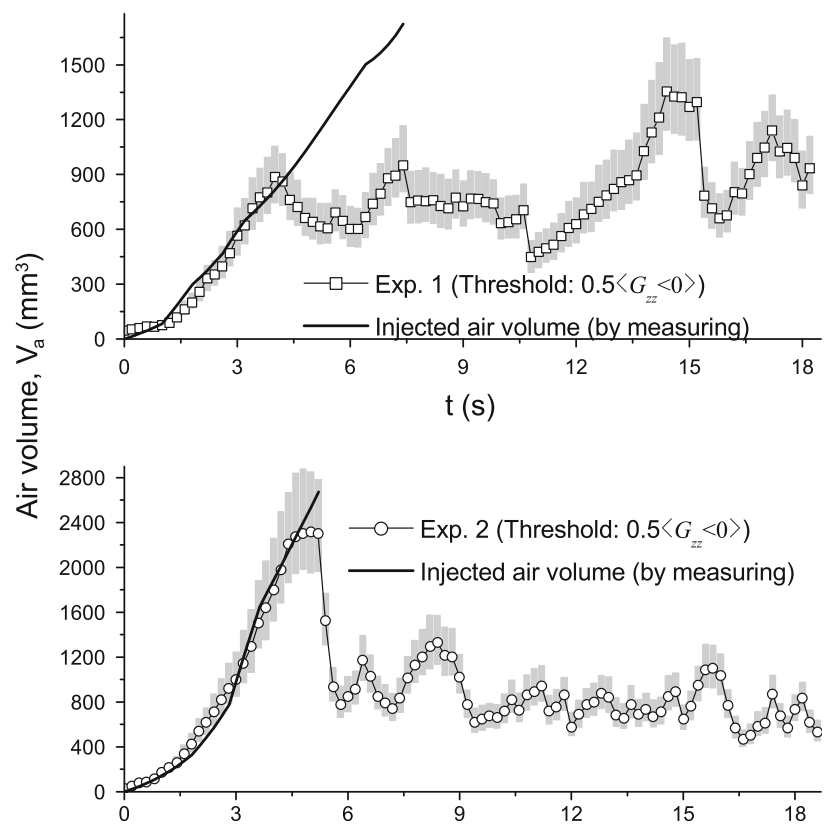

Fig. 14 Comparison of the injected volume (continuous line) with the volume calculated from the reconstructed plume structure in time using the "curvature" method, where the symbols refer to the selected threshold of $0.5\left\langle G_{z z}<0\right\rangle$ and the shaded columns represent a variation of $\pm 0.15\left\langle G_{z z}<0\right\rangle$

agreement between the two curves is quite good and overall better than the results from the other three methods. Figure $10 \mathrm{e}, \mathrm{j}$ depict the reconstructions of the plume structure, where the selected threshold is $0.5\left\langle G_{z z}<0\right\rangle$. Comparing Fig. 10e with Fig. 10a, we note that the two are almost identical. All the visible objects are preserved. From the side views Fig. 10e, j, one can see that the streaks associated with multiple exposures were satisfactorily removed. Additionally, the structure in Fig. 10e, j keeps connections between coherent elements of the plume, which are very important during the growth of the plume.

Since we have learnt that the "curvature" method performs quite well in terms of the morphology and volume of the reconstructed plume structure, we now use this method to show the evolution of the air plume by means of three

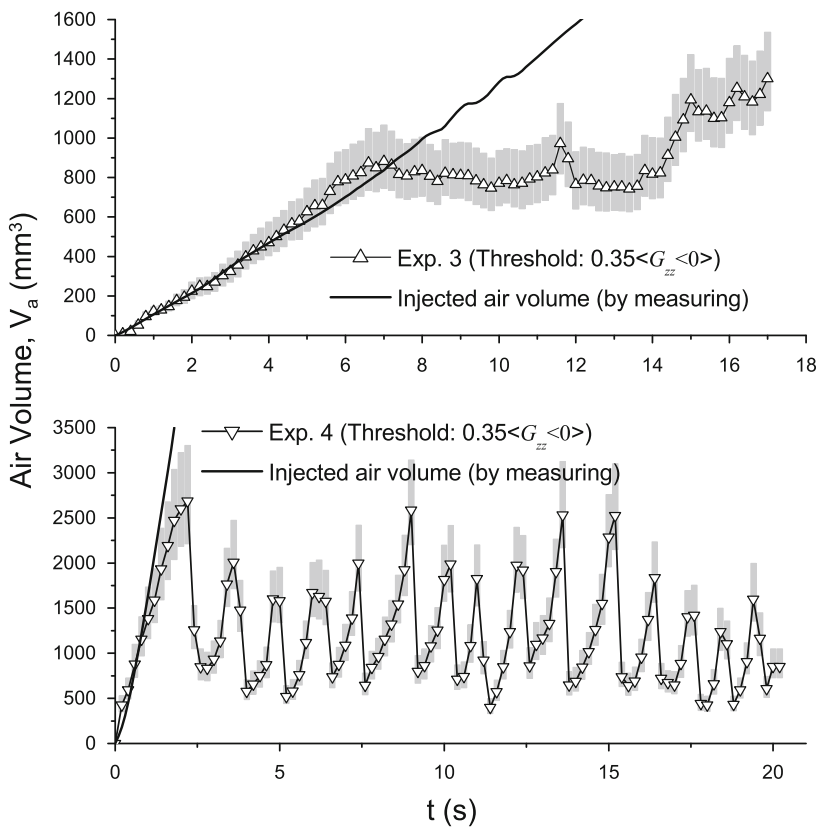

Fig. 16 Comparison of the injected volume (continuous line) with the volume calculated from the reconstructed plume structure in time using the "curvature" method, where the symbols refer to the selected threshold of $0.35\left\langle G_{z z}<0\right\rangle$ and the shaded columns represent a variation of $\pm 0.15\left\langle G_{z z}<0\right\rangle$

representative time instances, which are shown in Fig. 15. The structure appears to be more coherent when the experiment is started and more elongated and fragmented at later times. Also from this example of a time evolution, one can conclude that the reconstruction is morphologically consistent, in the sense that the dynamic growth of the main structures can be successfully recovered.

Finally, we further investigate the influence of the grain size on the "curvature" method. The experiments, Exp. 3 and Exp. 4, were performed using the medium consisting of finer grain $d_{2}$. Figure 16 shows the comparison between the injected air volume and the one calculated from the remaining structures after the "curvature" method was applied to experiments Exp. 3 and Exp. 4. Compared to coarser grain, a smaller coefficient of the threshold is
Fig. 15 3D reconstruction of the plume using the "curvature" method with the selected threshold is $0.5\left\langle G_{z z}<0\right\rangle$ for three time instances, $t=1,3$, and $5 \mathrm{~s}$. The injection rate in this experiment is $Q=41.1 \mathrm{ml} / \mathrm{min}$

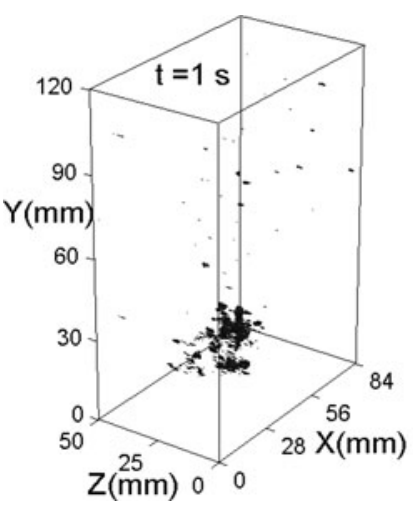

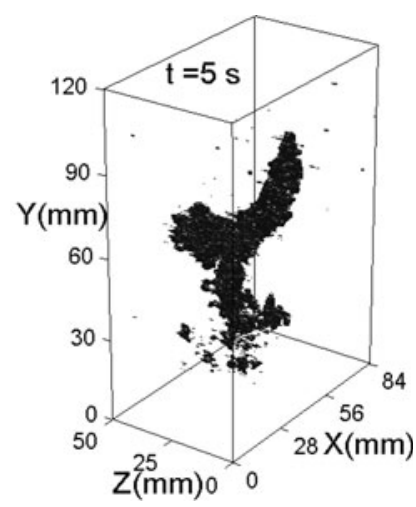


applied for the finer grain. The two curves are in correspondence with each other for Exp. 3 before $t=7.0$ and for Exp. 4 before $t=2.0 \mathrm{~s}$. For these two experiments, breakthrough occurs at $t=12.5 \mathrm{~s}$ and $t=2.3 \mathrm{~s}$, respectively, for Exp. 3 and Exp. 4. Similar to what we found in Exp. 1 and Exp. 2, air volume is lost due to the smooth interface that causes the reflection to be specular. Therefore, only the partial interface of the plume is visible in the camera. Two reconstructed air plumes at different time instances, $t=6.0 \mathrm{~s}$ of Exp. 3 and $t=2.0 \mathrm{~s}$ of Exp. 4, are shown in Fig. 17. It is well visible that the streaks related to multiple exposures were sufficiently removed.

\section{Conclusions}

In summary, we applied tomographic laser scanning and high-speed imaging to reconstruct the structure of an air plume injected into a liquid-saturated deformable porous medium. The medium is index matched by using a glycerinwater mixture as wetting fluid. The temporal resolution is $0.1 \mathrm{~s}$, and the spatial resolution is $0.17 \mathrm{~mm}$ in plane and about $1 \mathrm{~mm}$ out of plane of the laser sheet. Morphological consistency was tested by comparing the reconstructed plume with a photograph. However, the plume structure is found to be multiply exposed to some degree. This is because the light sheets overlap with each other between subsequent exposures and the reflection effects. Therefore, data reduction methods have to be applied before 3D reconstruction. Four image processing methods are applied and compared to each other: (i) thresholding of the grayvalue, (ii) erosion of the plume boundary, (iii) a "two-step" method that controls both the grayvalue and the object size of the images, and (iv) a "curvature" method that is based
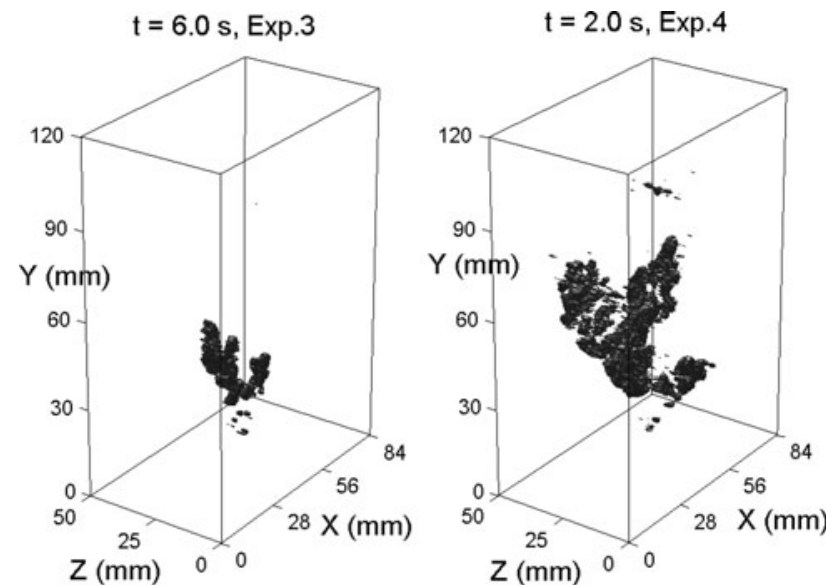

Fig. $173 \mathrm{D}$ reconstruction of the plume using the "curvature" method with the selected threshold is $0.35\left\langle G_{z z}<0\right\rangle$ for the time instance $t=6.0 \mathrm{~s}$ and $t=2.0 \mathrm{~s}$, respectively, for $Q=8.0 \mathrm{ml} / \mathrm{min}$ and $Q=141.7 \mathrm{ml} / \mathrm{min}$ on a threshold on the grayvalue curvature in scanning direction. These methods are validated, firstly, through comparison between the volume calculated from the reconstructed plume structure and the measured injected volume over time and, secondly, through comparison of the shapes obtained by the reconstruction. Compared to the other three methods, the "curvature" method shows a much better performance with respect to both criteria. It was found that our method performs well, when the interface of the plume is rough, whereas it has limitations when the interface becomes rather smooth. The outcome of this work will be the basis for future detailed investigations of growth characteristics of injected air plumes, such as breakthrough, surface-area-to-volume ratio, and fracture development.

Acknowledgments We thank Toni Blunschi for his help with the experimental setup, and Beat Lüthi and Klaus Hoyer for their helpful suggestions and discussions. This work is supported by ETH Research Grant TH-2606-1.

\section{References}

Anderson AL, Abegg F, Hawkins JA, Duncan ME, Lyons AP (1998) Bubble populations and acoustic interaction with the gassy floor of Eckernförde Bay. Cont Shelf Res 18:1807

Bensimon D, Kadanoff LP, Liang SD, Shraiman BI, Tang C (1986) Viscous flows in 2 dimensions. Rev Mod Phys 58:977-999

Best AI, Tuffin MD, Dix JK, Bull JM (2004) Tidal height and frequency dependence of acoustic velocity and attenuation in shallow gassy marine sediments. J Geophys Res 109:B08101

Best AI, Richardson MD, Boudreau BP, Judd AG, Leifer I, Lyons AP, Martens CS, Orange DL, Wheeler SJ (2006) Shallow seabed methane gas could pose coastal hazard. Eos Trans AGU 87(22):213-217

Boudreau BP, Gardiner BS, Johnson BD (2002) Rate of growth of isolated bubbles in sediments with a distributed source of methane. Limnol Oceanogr 46:616-622

Boudreau BP, Algar C, Johnson BD, Croudace I, Reed A, Furukawa Y, Dorgan KM, Jumars PA, Grader AS (2005) Bubble growth and rise in soft sediments. Geol Ore Depos 33(6):517-520

Brücker CH (1996) 3-D scanning-particle-image-velocimetry: technique and application to a spherical cap wake flow. Appl Sci Res 56(2-3): 157-179

Brücker CH (1997) 3-D scanning PIV applied to air flow in a motored engine using digital high speed video. Meas Sci Technol 8:1480-1492

Canler JP, Perret JM (1994) Biological aerated filters: assessment of the process based on 12 sewage treatment plants. J M Water Sci Technol 29(10-11):13-22

Chen MR, Hinkley RE, Killough JE (1996) Computed tomography imaging of air sparging in porous media. Water Resour Res 32:3013-3024

Chen Q, Kinzelbach W (2002) An NMR study of single- and twophase flow in fault gouge filled fractures. J Hydrol 259:236-245

Chevalier C, Lindner A, Clement E (2007) Destabilization of a Saffman-Taylor fingerlike pattern in a granular suspension. Phys Rev Lett 99:174501

Chevalier C, Lindner A, Leroux M, Clement E (2009) Morphodynamics during air injection into a confined granular suspension. J NonNewton Fluid Mech 158:63-72 
Dror I, Berkowitz B, Gorelick SM (2004) Effects of air injection on flow through porous media: observations and analyses of laboratory-scale processes. Water Resour Res 40:W09203

Fontenot MM, Vigil RD (2002) Pore-scale study nonaqueous phase liquid dissolution in porous media using laser-induced fluorescence. J Colloid Interface Sci 247(2):481-489

Fry VA, Selker JS, Gorelick SM (1997) Experimental investigations for trapping oxygen gas in saturated porous media for In situ bioremediation. Water Resour Res 33(12):2687-2696

Gardiner BS, Boudreau BP, Johnson BD (2003) Growth of diskshaped bubbles in sediments. Geochim Cosmochim Acta 67:1485-1494

Homsy GM (1987) Viscous fingering in porous media. Annu Rev Fluid Mech 19:271

Honkanen M (2006) Direct optical measurement of fluid dynamics and dispersed phase morphology in multiphase flows. $\mathrm{PhD}$. thesis, Tampere University of Technology, Tampere

Hoyer K, Holzner M, Lüthi B, Guala M, Liberzon A, Kinzelbach W (2005) 3D scanning particle tracking velocimetry. Exp Fluids 39:923-934

Johnson BD, Boudreau BP, Gardiner BS, Maass R (2002) Mechanical response of sediments to bubble growth. Mar Geol 187:347-363

Kong XZ, Kinzelbach W, Stauffer F (2009) Migration of air channels: an instability of air flow in mobile saturated porous media. Chem Sci Eng 64:1528-1535

Kong XZ, Kinzelbach W, Stauffer F (2010) Compaction and size segregation in a liquid-saturated grain packing due to pulsation effect during air injection. Chem Sci Eng 65(9):2680-2688

Knudsen HA, Sandnes B, Flekkoy EG, Maloy KJ (2008) Granular labyrinth structures in confined geometries. Phys Rev E 77:021301

Leeson A, Johnson PC, Johnson RL, Vogel CM, Hinchee RE, Marley M, Peargin T, Bruce CL, Amerson IL, Coonfare CT, Gillespie RD, Mc Whorter DB (2001) Air sparging, design and paradigm. Battelle, Columbus
Lenormand R, Zarcone C, Sarr A (1983) Mechanisms of the displacement of one fluid by another in a network of capillary ducts. J Fluid Mech 135:337-353

Lindner A, Bonn D, Poire EC, BenAmar M, Meunier J (2002) Viscous fingering in non-Newtonian fluids. J Fluid Mech 469:237-256

Montemagno CD, Gray WG (1995) Photoluminescent volumetric imaging-A technique for exploration of multiphase flow and transport in porous media. Geophys Res Lett 22(15):425-428

Oswald SE, Spiegel MA, Kinzelbach W (2007) Three-dimensional saltwater-freshwater fingering in porous media: contrast agent MRI as basis for numerical simulations. Magn Reson Imaging 25:537-540

Ovdat H, Berkowitz B (2006) Pore-scale study of drainage displacement under combined capillary and gravity effects in indexmatched porous media. Water Resour Res 42:W06411

Paull CK, Ussler W, Dallimore SR, Blasco SM, Lorenson TD, Melling H, Medioli BE, Nixon FM, McLaughlin FA (2007) Origin of pingo-like features on the Beaufort Sea shelf and their possible relationship to decomposing methane gas hydrates. Geophys Res Lett 34:L01603

Rashidi M, Peurrung L, Tompson A, Kulp TY (1996) Experimental analysis of pore-scale flow and transport in porous media. Adv Water Resour 19:163-180

Reddy KR, Semer R, Adams JA (1999) Air flow optimization and surfactant enhancement to remediate toluene-contaminated saturated soils using air sparging. Environ Manage Health 10(1):52-63

Stöhr M, Roth K, Jahne B (2003) Measurement of 3D pore-scale flow in index-matched porous media. Exp Fluids 35:159-166

Viotti P, Eramo B, Boni MR, Carucci A, Leccese M, Sbaffoni S (2002) Development and calibration of a mathematical model for the simulation of the biofiltration process. Adv Environ Res 7:11-33

Walmann T (1992) Visualization of transport phenomena in threedimensional porous media. M.Sc. thesis, University of Oslo, Oslo 